\title{
Coupled Personalisation of Electrophysiology Models for Simulation of Induced Ischemic Ventricular Tachycardia
}

\author{
J. Relan ${ }^{1}$, P. Chinchapatnam ${ }^{2}$, M. Sermesant ${ }^{1,2, \star}$, K. Rhode ${ }^{2}$, \\ H. Delingette ${ }^{1}$, R. Razavi ${ }^{2}$, and N. Ayache $^{1}$ \\ 1 Asclepios Research Project, INRIA, Sophia Antipolis, France \\ 2 Division of Imaging Sciences, St. Thomas Hospital, King's College London, UK \\ maxime.sermesant@sophia.inria.fr
}

\begin{abstract}
Despite recent efforts in cardiac electrophysiology modelling, there is still a strong need to make macroscopic models usable in planning and assistance of the clinical procedures. This requires model personalisation i.e. estimation of patient-specific model parameters and computations compatible with clinical constraints. Fast macroscopic models allow a quick estimation of the tissue conductivity, but are often unreliable in prediction of arrhythmias. On the other side, complex biophysical models are quite expensive for the tissue conductivity estimation, but are well suited for arrhythmia predictions. Here we present a coupled personalisation framework, which combines the benefits of the two models. A fast Eikonal (EK) model is used to estimate the conductivity parameters, which are then used to set the parameters of a biophysical model, the Mitchell-Schaeffer (MS) model. Additional parameters related to Action Potential Duration (APD) and APD restitution curves for the tissue are estimated for the MS model. This framework is applied to a clinical dataset provided with an hybrid X-Ray/MR imaging on an ischemic patient. This personalised MS Model is then used for in silico simulation of clinical Ventricular Tachycardia (VT) stimulation protocol to predict the induction of VT. This proof of concept opens up possibilities of using VT induction modelling directly in the intervention room, in order to plan the radio-frequency ablation lines.
\end{abstract}

\section{Introduction}

Cardiac arrhythmias are increasingly being treated by Radio-Frequency (RF) ablation procedures. These procedures still have unsatisfactory success rates of only $30-60 \%$ for VT, due to non availability of clinical consensus on optimum RF ablation patterns [1. There is still a need for substantial guidance in locating the optimum ablation lines. This guidance can be provided by personalised in silico cardiac electrophysiology models. Personalisation means estimation of the patient-specific model parameters which best fit the clinical data. It is required

\footnotetext{
* Corresponding author.
} 
to reveal hidden properties of the tissue and to develop predictive models that can be used to improve therapy planning and guidance. There are a variety of cardiac EP models developed at various scales. These models can be broadly categorised into three main categories: Biophysical Models (BM), Phenomenological Models (PM) and Eikonal Models (EM). BM [2] model ionic currents and are the most accurate and complex but hardly suitable for parameter estimation from clinical data. PM [3] are based on PDEs and mimic only the shape of action potential and are on intermediate level. EM [4] describes only the time at which a depolarisation wave reaches a given point. They can be very fast in computation [5], but less reliable in arrhythmia predictions due to the complexity of both refractoriness and curvature. To introduce models directly in the intervention room, the requirements are a low computational complexity, fast estimation of parameters and reliable predictions. These attributes cannot be found in one single model, thus here we present a new approach, where we combine two models to obtain these attributes and show an application to a clinical dataset. We also show how such personalised model can then be used to simulate in silico a clinical VT Stimulation protocol and can be potentially used to plan optimum RF ablation lines. In this paper, we present a coupled personalisation framework, which is fast and combines the benefits of an Eikonal (EK) model with those of a simplified biophysical model, the Mitchell-Schaeffer (MS) model. The fast 3D EK model is used to estimate the tissue conductivity parameter over the ventricles from the non-contact mapping of endocardial surface potential, using an adaptive iterative algorithm. This is then used to set the conductivity parameter of the 3D MS model. Additional parameters related to APD and APD restitution property of the tissue are then estimated locally using directly the 3D MS model. This framework is applied to a clinical data of an ischemic patient, containing of MR data for geometry and scar detection and electrophysiological data obtained from non-contact mapping. This data is obtained using Hybrid X- ray/magnetic resonance (XMR) suites [6]. The personalised 3D MS model is then used to simulate a clinical VT-Stim procedure to show a potential application of VT induction modelling.

\section{Simulation of Electrophysiology Models}

Anisotropic Fast Marching Model (EK Model): The EK model simulates the propagation of the depolarization wave in quiescent tissue, ignoring repolarisation phase. The EK model is governed by eikonal-diffusion (ED) equation and is based on Fast Marching Method (FMM), it can be written as

$$
c_{0} \sqrt{D(x)}\left(\sqrt{\nabla T(x)^{t} \mathbf{M} \nabla T(x)}\right)-\nabla \cdot(D(x) \mathbf{M} \nabla T(x))=\tau(x)
$$

where the superscript $t$ denotes transpose, $c_{0}$ is a dimensionless constant, and $\tau(x)$ is the cell membrane time constant. $D(x)$ is the square of the tissue space constant along the fiber and is related to the specific conductivity of the tissue in the fiber direction. The anisotropicity is incorporated in the Diffusion tensor and 
is given by $\mathbf{M}$. The nonlinear term (Eq1) is solved using a fixed point iterative method combined with a very fast eikonal solver as explained in [7.

Biophysical Model (MS Model): The MS model [8] is a 2-variable simplified biophysical model derived from the 3 -variable Fenton Karma (FK) ionic model [9]. It models the transmembrane potential as the sum of a passive diffusive current and several active reactive currents including a sodium ion (influx) current and a potassium ion (outflux) current. Unlike FK model, it does not model the Calcium ion current. The MS model is described by the following system of Ordinary Differential Equations (ODE),

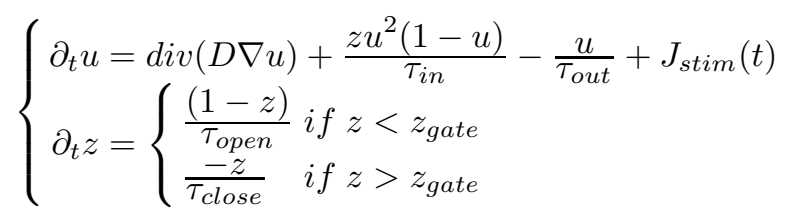

where, $u$ is a normalised transmembrane potential variable, and $z$ is a gating variable for sodium ion influx which makes the gate open and close, thus depicting the depolarisation and repolarisation phase. $J_{i n}=\left(z u^{2}(1-u)\right) / \tau_{\text {in }}$ represents the inward sodium ion current which raises the action potential voltage and $J_{\text {out }}=-u / \tau_{\text {out }}$ represents the outward potassium ion current that decreases the action potential voltage describing repolarisation. $J_{\text {stim }}$ is the stimulation current, at the pacing location. The diffusion term in the model is controlled by the diffusion tensor $D$. This spatial diffusion can be related to a pseudoconductivity. In the longitudinal direction of the fibre, this pseudo-conductivity is set to $d$ which is one of the parameters we adjust, and to $d / 2.5^{2}$ in the transverse directions. The electrophysiology model is solved spatially using P1 Finite Element Method (FEM), and in time using an semi-implicit scheme as Modified Crank-Nicolson/Adams-Bashforth (MCNAB) scheme, which is evaluated in terms of accuracy, stability and computational time [10.

\section{Coupled Personalisation Method}

Apparent Conductivity Parameter Estimation: Cardiac tissue conductivity is a crucial feature for the detection of conduction pathologies. The Apparent Conductivity (AC) of the tissue can be measured by the parameter $D(x)$ in the EK model. It is initially estimated on the endocardial surface as a global value using a simple bisection method which matches the average conduction velocity of the measured Depolarisation Time (DT) isochrones to the simulated ones. Using it as an initial guess, an adaptive multi-level zonal decomposition algorithm is used, which minimizes the mean-squared difference of the simulated and measured DT isochrones at each level using a Brent's Optimisation Algorithm presented in [5]. Due to the absence of transmural electrical propagation information, we assume no variation across the left ventricle myocardium (excluding LV endocardium and scars) and hence we prescribe a single value for the myocardial tissue across the LV wall. The AC values for RV endocardium 
and RV myocardial mass are set at $5.0 \mathrm{~mm}$ and $0.64 \mathrm{~mm}$ (from literature [4]). The LV myocardial AC value is estimated by one-dimensional minimisation of the following cost function (mean-squared difference of simulated and measured isochrones at endocardium + squared difference of simulated and measured QRS duration). The simulated QRS duration is calculated as the difference between the maximum and the minimum depolarisation times in the biventricular mesh and the measured QRS duration is estimated from the surface ECG.

Coupling of EK and MS Model Parameters: The AC parameter for EK model $d_{E K}(D(x)$ in Eq1) is a scale for the diffusion speed of the depolarisation wavefront in the tissue. The diffusion tensor used in Eq 1 is $\mathbf{M}=\mathbf{A} \overline{\mathbf{D}} \mathbf{A}^{t}$, where $\mathbf{A}$ is the matrix defining the fiber directions in the global coordinate system and $\overline{\mathbf{D}}=d_{E K} \operatorname{diag}\left(1, \lambda^{2}, \lambda^{2}\right) . \lambda$ is the anisotropic ratio of space constants transverse and along the fiber direction and is 0.4 in human myocardium 4 . The model Conduction Velocity $(\mathrm{CV})$ is related to $d_{E K}$ as,

$$
c_{E K}=\frac{c_{0} \sqrt{d_{E K}}}{\tau} \text { in } 1 \mathrm{D} \text { and } c_{E K}=\alpha_{E K} \sqrt{d_{E K}}+\beta_{E K} \text { in 3D }
$$

where the constants $\alpha_{E K}$ and $\beta_{E K}$ are introduced to take into account the curvature effect and numerical diffusion and discretization errors in $3 \mathrm{D}$. The corresponding conductivity parameter for MS model, $d_{M S}$ is also a scale for the wave diffusion speed in the tissue. The diffusion tensor $D$ used in Eq 2 is $D=\mathbf{A} \overline{\mathbb{D}} \mathbf{A}^{t}$, where $\mathbf{A}$ is the same as in EK model, but $\overline{\mathbb{D}}=d_{M S} \operatorname{diag}(1, r, r)$ with $r$ as conductivity anisotropy ratio in the transverse plane and is set to $\lambda^{2}$ as in EK model. The model $\mathrm{CV}$ here is related to $d_{M S}$ as,

$$
c_{M S} \propto \sqrt{\frac{d}{\tau_{i n}}} \text { in } 1 \mathrm{D} \text { and } c_{M S}=\alpha_{M S} \sqrt{d_{M S}}+\beta_{M S} \text { in 3D }
$$

where the constants $\alpha_{M S}$ and $\beta_{M S}$ are introduced for the same reasons as of EK model, while $\tau_{i n}$ is kept as a constant. The estimated AC parameter $d_{E K}$ can then be used to estimate the parameter $d_{M S}$. The parameter $d_{E K}$ gives model $\mathrm{CV} c_{E K}$, which is similar to the actual measured data $\mathrm{CV}\left(c_{m s d}\right)$ after the parameter estimation step. Thus to have MS model CV $\left(c_{M S}\right)$ similar to the measured data, it has to be similar to EK model CV $\left(c_{E K}\right)$. The constants $\alpha_{E K}$ and $\beta_{E K}$ represent numerical errors for EK model based on FMM. They are different from the constants $\alpha_{M S}$ and $\beta_{M S}$, which is based on FEM. These constants are determined in $3 \mathrm{D}$ for a mesh representing the slab of a tissue $\left(\left[\begin{array}{ll}0 & 10\end{array}\right]\right.$ $\times\left[\begin{array}{ll}0 & 10\end{array} \times\left[\begin{array}{ll}0 & 10\end{array}\right]\right)($ with a mean edge length of tetrahedra same as the ventricular mesh). We performed several simulations with various $d_{E K}$ and $d_{M S}$ values and noted the corresponding $c_{E K}$ and $c_{M S}$ values. Then, we fit the analytical curves given in Eq 3 \& 4 in least square sense and determine the constants. The constants estimated are $\alpha_{E K}=5.21, \beta_{E K}=0.07, \alpha_{M S}=3.12, \beta_{M S}=0.31$. Then, the personalised $d_{M S}$ values are computed from corresponding estimated $d_{E K}$ values using the condition, that $c_{m s d}=c_{E K}=c_{M S}$ after personalisation.

Parameter Estimation for APD: APD for a single heart cycle is defined by the model as, $A P D_{\max }=\tau_{\text {close }} \ln \left(1 / h_{\text {min }}\right)$ where $h_{\text {min }}=4\left(\tau_{\text {in }} / \tau_{\text {out }}\right)$ As 

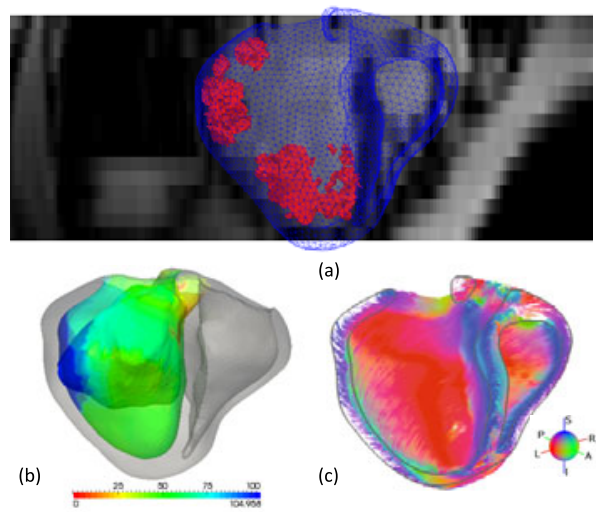

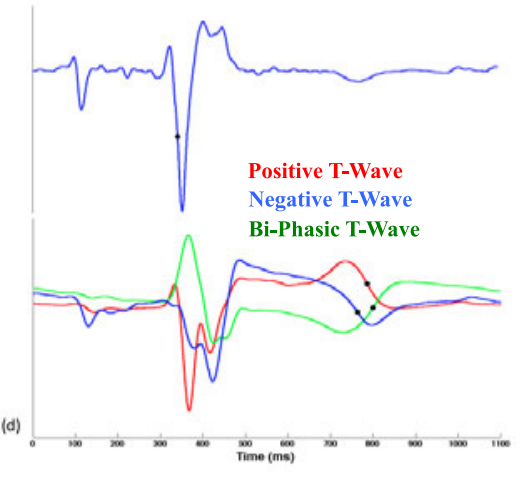

Fig. 1. (a) shows the MR data, segmented mesh with scars (in red), (b) shows XMR registration of Ensite LV surface with MR data mesh, with values projected from Ensite to MR LV surface,(c) shows the fibre orientation used, (d) shows the unipolar electrograms for detection (black dots) of depolarisation time (upper) and repolarisation time (lower) from positive(red), negative(blue) and biphasic(green) $\mathrm{T}$ waves

we have only one measured APD dependent on three parameters, We chose to estimate $\tau_{\text {close }}$, while keeping the other parameter values from the literature 8 . The reason is that $\tau_{\text {close }}$ has no sensitivity towards the conductivity parameter estimation [10], whereas $\tau_{\text {in }}$ and $\tau_{\text {out }}$ do have. This defined relationship remains valid also in $3 \mathrm{D}$ thus allowing us to directly estimate locally at each vertex, the parameter $\tau_{\text {close }}$ without model simulations. To have a smooth gradation of APD from epicardium to endocardium, we diffuse the $\tau_{\text {close }}$ values spatially in the LV myocardium from Endocardium to Epicardium. For RV, we fix one value measured from the QT interval given through surface ECG.

Parameter Estimation for APD Restitution : APD Restitution is a property of a cardiac cell and defines its refractoriness. It is also heterogeneously distributed. It is a relationship between of the next cycle APD and the Diastolic Interval (DI) of the previous cycle. The slope of these restitution curves is controlled by $\tau_{\text {open }}$ and depicts the APD heterogeneity present at multiple heart rates. APD restitution curve for MS model is explicitly derived as, $f\left(D I_{n}\right)=$

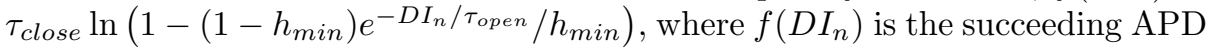
and $D I_{n}$ is the preceding DI at cycle $n$. Here, we use the $\tau_{\text {close }}$ values estimated as defined before, as it controls the APD at normal sinus rythm. And, the slope parameter, $\tau_{\text {open }}$ is estimated here with $h_{\text {min }}$ fixed with values from the literature 8]. Here we minimise a cost function which minimises the error between model predicted APD $\left(f\left(D I_{n}\right)\right)$ and actual measured APD $\left(A P D_{n+1}^{m s d}\right)$ for a number of pacing frequency, where $n$ is the cycle number. The Diastolic Inter$\operatorname{val}\left(D I^{m s d}\right)$ is measured from the data as $D I_{n}^{m s d}=1 / f-A P D_{n}^{m s d}$, where $f$ is the heart rate, detected from the ECG waveforms. The parameter optimisation method used here is a non-linear constrained Active-Set Algorithm, with constraints on $\tau_{\text {open }}$ to be in the range of literature values [8]. 


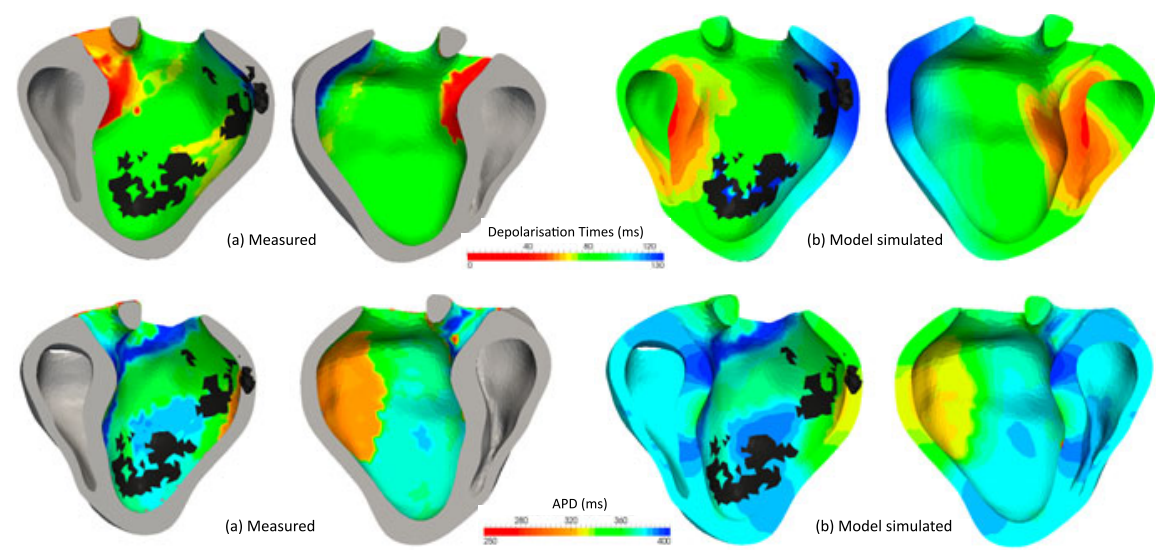

Fig. 2. Upper row shows the comparison of the measured Depolarisation Time (DT) isochrones on the LV surface only with model simulated DT isochrones on the whole heart, lower row shows the same for measured (LV surface only) and model simulated (whole heart) APD maps

\section{Application to Clinical Data}

The coupled personalisation framework is applied on a clinical data obtained during an electrophysiology study in the hybrid X-ray/MR environment. The electrical measurements obtained using the Ensite system were registered to the patient anatomy using XMR registration. The electrical data was collected with high pass filter settings for prominent QRS detection and with low pass filter for $\mathrm{T}$ Wave detection. The depolarisation times were detected from the $d V / d t_{\max }$ and $d^{2} V / d t^{2}$ of the unipolar electrograms $V$. And the repolarisation times were detected using $d V / d t_{\max }$ for the negative $\mathrm{T}$ wave, at the $d V / d t_{\text {min }}$ for the positive $\mathrm{T}$ wave, and the mean time between $d V / d t_{\max }$ and $d V / d t_{\text {min }}$ for the biphasic $\mathrm{T}$ waves. The data was collected from an ischemic patient at normal sinus rythm and 5 paced modes all at 100 beats per minute. The scars were segmented manually from the Delayed Enhancement MR data.

Estimated Parameters: The AC parameters estimated using EK model show a high conduction on the epicardium depicting the purkinje network and shows a conduction block near the scar as shown in Fig [3(a). The coupled MS model conductivity parameters are then estimated from AC. The mean absolute error on simulated depolarisation times with measured, after personalisation is $7.1 \mathrm{~ms}$ for EK model and $8.5 \mathrm{~ms}$ for $\mathrm{MS}$ model $(\approx 6-7 \%$ of depolarisation duration $(131 \mathrm{~ms}))$. The mean absolute error on APD is $8.71 \mathrm{~ms}(\approx 2 \%$ of APD $(300 \mathrm{~ms}))$, showing a good fit as well. Fig 3 (b) (white contour) shows the heterogeneity of the measured APD in terms of the estimated parameter $\tau_{\text {close }}$, as shorter on the lateral wall of the LV compared to the septum. Also near the scar and the region between the two scars (called isthmus) Fig 3 (b) (black contour), we have 


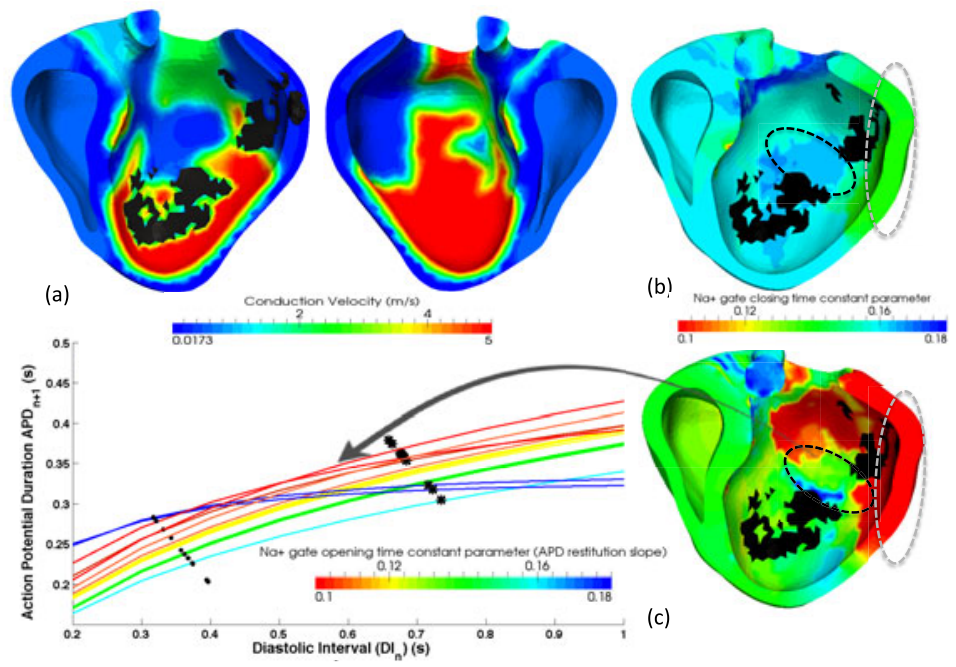

Fig. 3. (a) shows the conduction velocity estimated from AC maps, (b) shows the parameter $\tau_{\text {close }}$ estimated for APD, lower $\tau_{\text {close }}$ values has low measured APD (white contour) and vice versa, (c) shows the parameter $\tau_{\text {open }}$ estimated for APD restitution and the heterogeneity of the restitution curves for the isthmus (black contour), low $\tau_{\text {open }}$ values (red) have steep slopes \& high (blue) have flat slopes for restitution curves

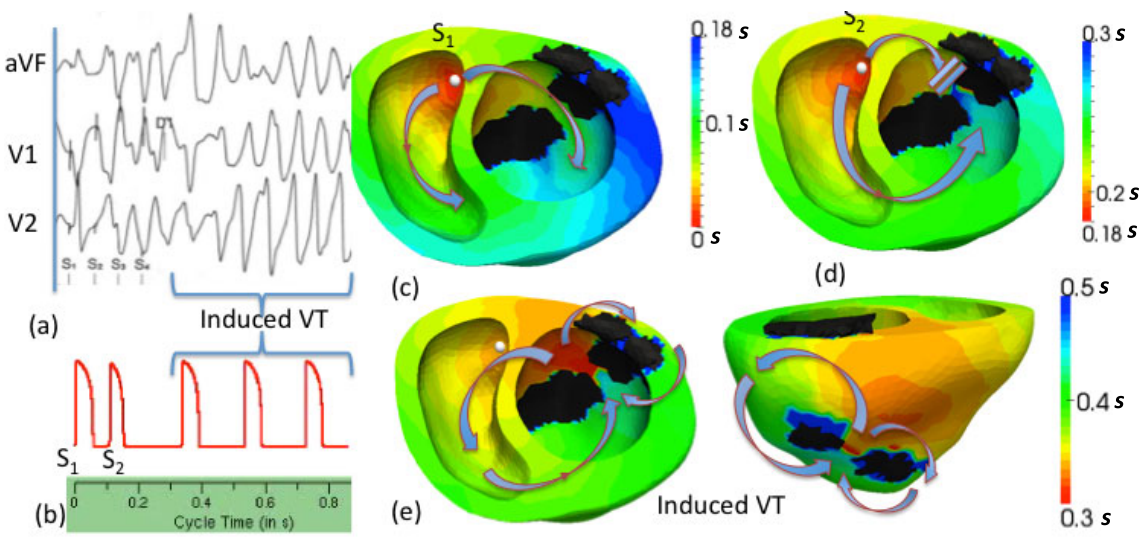

Fig. 4. (a) shows unipolar electrograms recorded for a clinical VT-Stim protocol, (b) shows the simulated protocol for two extrastimuli, with coupling interval of $100 \mathrm{~ms}$. (c) show DT isochrones(in s) for $S_{1}$ stimulus and (d) shows for $S_{2}$, we have a unidirectional block created in the isthmus. (e) shows DT isochrones for induced monomorphic VT.

a longer APD compared to the neighbours. For the APD Restitution, the mean absolute error after fitting the resitution curves is $1.13 \mathrm{~ms}$, showing a good fit also seen from the Fig 3(a). There is a APD restitution heterogeneity present for the lateral and septal walls as shown in Fig [3(c). Isthmus has flatter restitution 
slopes compared to the neighbours, thus having a longer refractory period and causing a unidirectional re-entry as seen in VT-Stim procedure.

Ischemic Ventricular Tachycardia Stimulation: Programmed ventricular stimulation is a clinical protocol and consists of a number of extra stimuli introduced at two ventricular sites (RV-Apex \& RV-Outflow tract), using various Cycle Lengths (CL), with varying coupling interval. This protocol is tested directly on the patient, without any planning, to collect information about the $\mathrm{VT}$ and to plan the RF ablation lines. It may be time consuming or fail, when VT is not inducible and recurrent. We use the personalised 3D MS model of the ischemic patient data to simulate in silico this protocol. Here we follow a conventional VT-Stim protocol with RV-Apex pacing site, 2 extrastimuli and a shortest coupling interval of $100 \mathrm{~ms}$ at $600 \mathrm{~ms}$ pacing cycle length. The results on inducibility are shown in Fig 4 and the causes of rentry were pacing location, restitution heterogeneity in isthmus compared to healthy Fig 3(c) and slow conductivity near the scars.

\section{Conclusion}

This novel approach of coupling models for fast estimation of hidden parameters related to the cardiac tissue such as conductivity, APD and APD restitution could enable the clinical use of cardiac electrophysiology models. The parameter estimation algorithm is used on a real interventional data and the obtained results are very encouraging. The estimated conductivity, APD and APD restitution parameters are able to identify the healthy areas from the pathological ones (scar and isthmus). The personalised MS model was able to simulate a clinical VT-Stim protocol in order to assess the risk of VT and fibrillation. This opens up possibilities of introducing patient-specific models in clinics to provide aid in treatment and planning of RF ablation procedures. In future, we need to evaluate the prediction ability of the personalised models for arrhythmias.

Acknowledgements. The research leading to these results was partially supported by a Prize from Microsoft Research Cambridge and by the EUHeart project (FP7/2007-2013 under grant agreement n 224495).

\section{References}

1. Aliot, E., Stevenson, W., Almendral-Garrote, J.: Ehra/hrs expert consensus on catheter ablation of ventricular arrhythmias. Europace (2009)

2. Noble, D.: Modeling the heart. Physiology (2004)

3. FitzHugh, R.: Impulses and physiological states in theoretical models of nerve membrane. Biophysical Journal (1961)

4. Keener, J., Sneyd, J.: Mathematical Physiology. Springer, Heidelberg (1998)

5. Chinchapatnam, P., Rhode, K., Ginks, M., Rinaldi, C., Lambiase, P., Razavi, R., Arridge, S., Sermesant, M.: Model-based imaging of cardiac apparent conductivity and local conduction velocity for diagnosis and planning of therapy. IEEE Trans. on Medical Imaging 27 (2008) 
6. Rhode, K., Sermesant, M., Brogan, D., Hegde, S., Hipwell, J., Lambiase, P., Rosenthal, E., Bucknall, C., Qureshi, S., Gill, J., Razavi, R., Hill, D.: A system for real-time XMR guided cardiovascular intervention. IEEE TMI 24 (2005)

7. Sermesant, M., Konukoglu, E., Delingette, H., Coudiere, Y., Chinchaptanam, P., Rhode, K., Razavi, R., Ayache, N.: An anisotropic multi-front fast marching method for real-time simulation of cardiac electrophysiology. In: Sachse, F.B., Seemann, G. (eds.) FIHM 2007. LNCS, vol. 4466, pp. 160-169. Springer, Heidelberg (2007)

8. Mitchell, C.C., Schaeffer, D.G.: A two current model for the dynamics of cardiac membrane. B. of Mathematical Biology (2003)

9. Fenton, F., Karma, A.: Vortex dynamics in three-dimensional continuous myocardium with fiber rotation: Filament instability and fibrillation. In: CHAOS (1998)

10. Relan, J., Sermesant, M., Delingette, H., Pop, M., Wright, G.A., Ayache, N.: Quantitative comparison of two cardiac electrophysiology models using personalisation to optical and mr data. In: ISBI (2009) 